\title{
BMJ Open Accuracy and consequences of using trial-of-antibiotics for TB diagnosis (ACT-TB study): protocol for a randomised controlled clinical trial
}

\author{
Titus Henry Divala (D) , ${ }^{1,2,3}$ Katherine L Fielding, ${ }^{1,4}$ Derek J Sloan, ${ }^{5}$ Neil French, ${ }^{6}$ \\ Marriott Nliwasa, ${ }^{1,2}$ Peter MacPherson, ${ }^{3,7}$ Chikondi Charity Kandulu, ${ }^{2,3}$ \\ Lingstone Chiume, ${ }^{2,3}$ Sanderson Chilanga, ${ }^{2,3}$ Masiye John Ndaferankhande, ${ }^{3}$ \\ Elizabeth L Corbett ${ }^{1,2,3}$
}

To cite: Divala TH, Fielding KL, Sloan DJ, et al. Accuracy and consequences of using trialof-antibiotics for TB diagnosis (ACT-TB study): protocol for a randomised controlled clinical trial. BMJ Open 2020;10:e033999. doi:10.1136/ bmjopen-2019-033999

- Prepublication history and additional material for this paper are available online. To view these files, please visit the journal online (http://dx.doi. org/10.1136/bmjopen-2019033999).

Received 01 September 2019 Revised 20 February 2020 Accepted 27 February 2020

Check for updates

(C) Author(s) (or their employer(s)) 2020. Re-use permitted under CC BY-NC. No commercial re-use. See rights and permissions. Published by BMJ.

For numbered affiliations see end of article.

Correspondence to

Dr Titus Henry Divala;

titus.divala@Ishtm.ac.uk

\section{ABSTRACT}

Introduction Over $40 \%$ of global tuberculosis case notifications are diagnosed clinically without mycobacteriological confirmation. Standard diagnostic algorithms include 'trial-of-antibiotics'-empirical antibiotic treatment given to mycobacteriology-negative individuals to treat infectious causes of symptoms other than tuberculosis, as a 'rule-out' diagnostic test for tuberculosis. Potentially 26.5 million such antibiotic courses/year are prescribed globally for the 5.3 million/ year mycobacteriology-negative patients, making trial-ofantibiotics the most common tuberculosis diagnostic, and a global-scale risk for antimicrobial resistance (AMR). Our systematic review found no randomised controlled trial (RCT) to support use of trial-of-antibiotic. The RCT aims to determine the diagnostic and clinical value and AMR consequences of trial-of-antibiotics.

Methods and analysis A three-arm, open-label, RCT randomising (1:1:1) Malawian adults ( $\geq 18$ years) seeking primary care for cough into: (a) azithromycin $500 \mathrm{mg}$ one time per day for 3 days or (b) amoxicillin $1 \mathrm{~g}$ three times per day for 5 days or (c) standard-of-care (no immediate antibiotic). We will perform mycobacteriology tests (microscopy, Xpert MTB/RIF (Mycobacterium tuberculosis/rifampicin) and Mycobacterium tuberculosis culture) at baseline. We will use audiocomputer-assisted self-interview to assess clinical improvement at day 8 . First primary outcome will be proportion of patients reporting day 8 improvement out of those with negative mycobacteriology (specificity). Second primary outcome will be day 29 incidence of a composite endpoint of either death or hospitalisation or missed tuberculosis diagnosis. To determine AMR impact we compare proportion of resistant nasopharyngeal Streptococcus pneumoniae isolates on day 29. 400 mycobacteriology-negative participants/arm will be required to detect $\mathrm{a} \geq 10 \%$ absolute difference in diagnostic specificity with $80 \%$ power. We will estimate measures of effect by comparing outcomes in antibiotic arms (combined and individually) to standard-of-care.

Ethics and dissemination The study has been reviewed and approved by Malawi College of Medicine Research and Ethics Committee, London School of Hygiene \&

\section{Strengths and limitations of this study}

- To our knowledge this is the first randomised controlled trial to address benefits and consequences of using antibiotics as an exclusion diagnostic for tuberculosis, a widely used practice that results in millions of antibiotic prescriptions/year.

- We will also contribute evidence on antimicrobial resistance affecting common antimicrobials used for managing respiratory infections.

- The use of audio computer-assisted self-interview for assessing clinical response and adherence to antibiotic treatment which can be used in future studies.

- Acknowledged weaknesses include limited power to evaluate safety of deferred antibiotic treatment, conduct subgroup analysis by HIV status and the possibility that participants randomised to the standard-of-care arm may find alternative access to antibiotics therefore misclassifying exposure/intervention status.

Tropical Medicine (LSHTM) Research Ethics Committee and Regional Committee for Health and Research Ethics - Norway, and Malawi Pharmacy, Medicines and Poisons Board. We will present abstracts at relevant conferences, and prepare a manuscript for publication in a peerreviewed journal.

Trial registration number The clinical trial is registered with ClinicalTrials.gov, NCT03545373

\section{INTRODUCTION}

The high case-fatality rate for tuberculosis, the leading global infectious cause of death in adults ${ }^{1}$ with approximately 10 million cases and 1.6 million deaths in $2017,{ }^{2}$ in part reflects suboptimal diagnostics. ${ }^{3-6}$ To complement this diagnostic gap, standard algorithms throughout the world include a 'trial-of-antibiotics' (figure 1). This is a course of broad-spectrum antibiotics, with negligible 


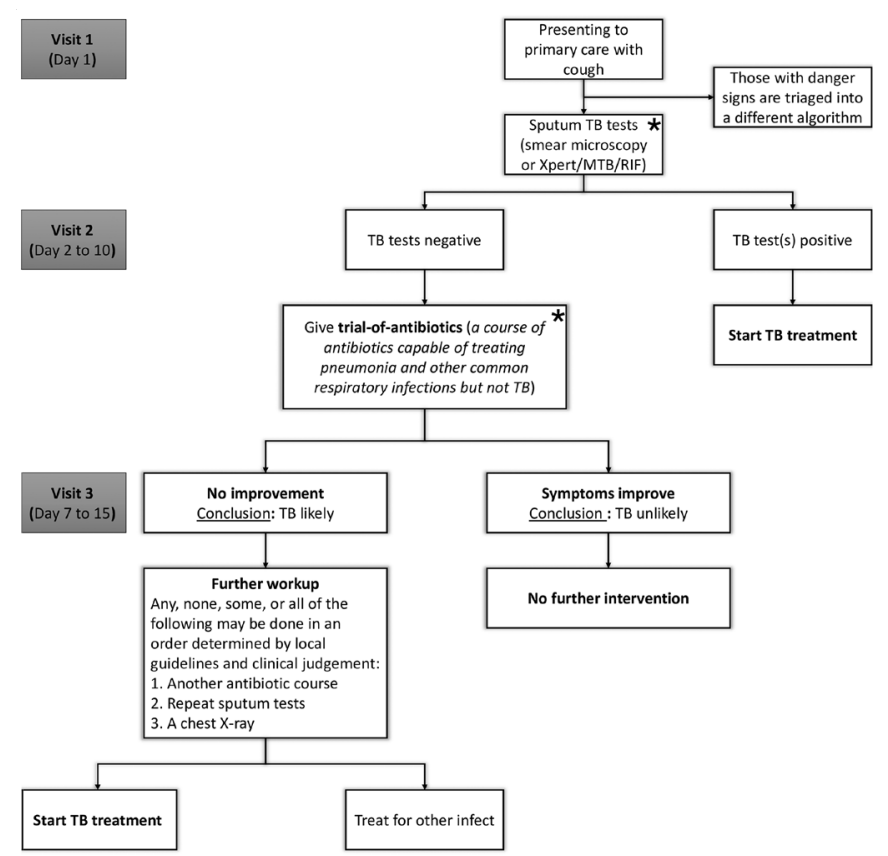

Figure 1 The position of trial-of-antibiotics in standard algorithms for diagnosis of tuberculosis in low- and middleincome countries (based on the 2018 WHO GLI model guidelines and as implemented in national guidelines, for example, Ghana, Malawi and South Africa). ${ }^{*}$ The common clinical practice is that outpatients start antibiotics at the time of submitting sputum, to avoid the need for a third clinic visit to complete the algorithm. GLI,Global Laboratory Initiative; MTB, Mycobacterium tuberculosis; RIF, rifampicin; TB, tuberculosis.

Mycobacterium tuberculosis activity, given to patients with symptoms such as cough in order to 'rule-out' or 'rule in' tuberculosis. ${ }^{7-9}$ In clinical practice and most national guidelines (summarised in figure 1), patients who have negative sputum mycobacteriology and have responded to antibiotic treatment are considered tuberculosis-negative while those who remain symptomatic are deemed likely to have tuberculosis and undergo further evaluations potentially leading on to receiving tuberculosis treatment. ${ }^{7-9}$

We estimate that 26.5 million courses of antibiotics are prescribed in the diagnosis of the 5.3 million smearnegative tuberculosis registrations recorded annually, ${ }^{10}$ making antibiotics the most common diagnostic for tuberculosis. ${ }^{11}$ Our 26.5 million estimate assumes that for every one smear-negative tuberculosis case detected, five antibiotics courses are used: the first two courses being given to patients are ultimately registered as smearnegative tuberculosis, while the other three courses represent patients whose symptoms resolved without starting anti-tuberculosis treatment. ${ }^{4}{ }^{12}$ This high frequency of prescription of important broad-spectrum antibiotics raises a global-scale risk for antimicrobial resistance (AMR) which like tuberculosis, is a major crisis, becoming in 2016 one of only four health topics ever to be discussed at the United Nations General Assembly. ${ }^{13-16}$

We performed a systematic literature review ${ }^{17}$ which demonstrated that, despite being in global and national guidelines for decades, trial-of-antibiotics has a limited supporting evidence base but with the available evidence suggesting poor diagnostic performance. ${ }^{18}$ None of the identified studies was an randomised controlled trial (RCT) and most of the observational studies were very small and not primarily designed to assess the benefits and consequences of trial-of-antibiotics. Pooled sensitivity and specificity of trial-of-antibiotics versus mycobacteriology tests were below internationally defined minimum performance profiles for tuberculosis diagnostics. ${ }^{19}$

We hypothesise that use of antibiotics in the course of evaluating patients for tuberculosis has both benefits and risks that need to be weighed carefully to optimise patient and public health outcomes. We will address evidence gaps related to (a) accuracy, (b) antimicrobial resistance and (c) impact on clinical outcomes of trial-of-antibiotics by conducting an RCT (ACT-TB study) recruiting adult patients with cough presenting to health centres in Blantyre, Malawi. To our knowledge this is the first randomised controlled trial to rigorously address these questions.

\section{METHODS AND ANALYSIS \\ Study design}

This is a three-arm individually randomised (1:1:1), openlabel controlled clinical trial (RCT) investigating accuracy and broader clinical, and antimicrobial resistance impact of using trial-of-antibiotics to rule-out tuberculosis among adults presenting with cough at primary care centres in Malawi (figure 2). The trial is registered with ClinicalTrials.gov (online supplementary appendix 2). The full trial protocol is provided as online supplementary appendix 3.

\section{Study setting}

We will screen adults aged at least 18 years presenting to Limbe and Ndirande health centres in Blantyre, Malawi. Blantyre has an estimated tuberculosis prevalence of 1014 per 100000 (95\% CI: 486 to 1542), and an estimated adult HIV prevalence of $12.7 \%$ (95\% CI: 11.9 to 13.6$).{ }^{20}$

\section{Eligibility criteria}

We will offer enrolment to patients who satisfy the following inclusion and exclusion criteria.

\section{Inclusion criteria}

- Ambulatory clinic attendees presenting with cough.

- Unwell for at least 14 days.

- Aged at least 18 years.

- Reside in Blantyre and willing to return to the same clinic for follow-up visits over the entire study period.

\section{Exclusion criteria}

- Self-reported allergy to study medications.

- WHO/Malawi National Tuberculosis Programme danger signs: respiratory rate $>30 / \mathrm{min}$, temperature $>39^{\circ} \mathrm{C}$, heart rate $>120 /$ minute, confused/agitated, respiratory distress, systolic blood pressure $<90 \mathrm{~mm}$ $\mathrm{Hg}$, inability to walk unassisted. 


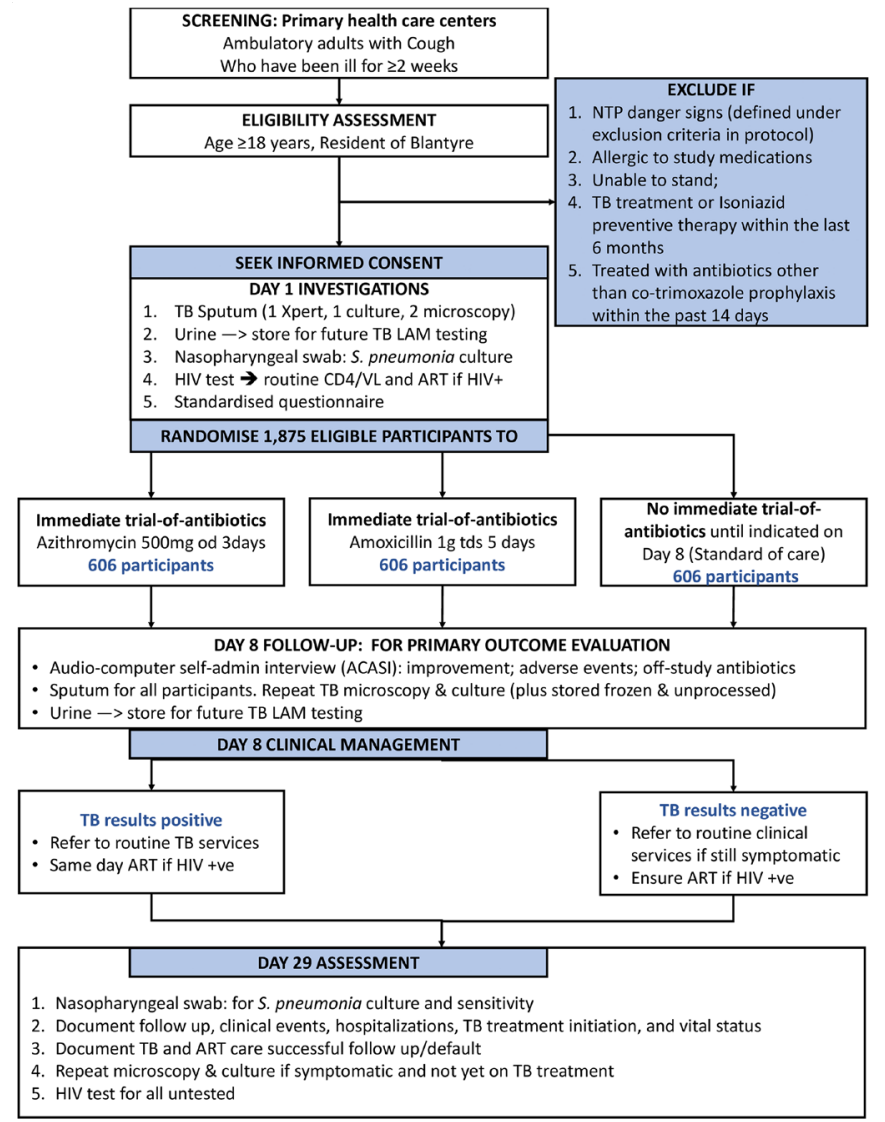

Figure 2 Flow diagram for the clinical trial in Blantyre, Malawi. ART;antiretroviral therapy; LAM, lipoarabinomannan; NTP, Malawi National Tuberculosis Programme; TB, tuberculosis; VL, viral load.

- Treated with antibiotics other than co-trimoxazole prophylaxis within the past 14 days.

- Tuberculosis treatment or isoniazid preventive therapy within the last 6 months.

\section{Interventions}

We will randomise participants, in a ratio of $1: 1: 1$, to the following arms:

- Arm 1 (azithromycin): Azithromycin $500 \mathrm{mg}$ taken one time per day for 3 days from enrolment day.

- Arm 2 (amoxicillin): Amoxicillin $1 \mathrm{~g}$ taken three times per day for 5 days from enrolment day.

- Arm 3 (standard of care): No study antibiotic prescription.

\section{Rationale for interventions}

Amoxicillin was chosen because it is the standard antibiotic used as first-line treatment and for trial-of-antibiotics in Malawi. However, amoxicillin may not demonstrate the best performance for trial-of-antibiotics because of increasing resistance, and a narrow coverage for aetiology of community acquired pneumonia and 'atypical' organisms. We chose azithromycin to represent the optimal biological specificity of an oral regimen due to more complete coverage of atypical organisms that cause community acquired pneumonia (eg, mycoplasma and chlamydia), and also the low resistance rates in Malawi where macrolides are rarely used. The dose for azithromycin is as recommended in the British National Formulary (BNF) as treatment for communityacquired pneumonia. ${ }^{21}$ The dose for amoxicillin is the $\mathrm{BNF}$ recommendation for severe infections but it is the recommended first-line established by the Department of Medicine at Queen Elizabeth Central Hospital (Blantyre, Malawi) based on local microbiology.

\section{Timing of interventions}

The standard of care in Malawi defined by National Tuberculosis Programme guidelines for primary care patients presenting with cough who are otherwise well (no danger signs) is to take two sputum specimens for smear microscopy or Xpert and ask patients to return for results, typically 3 days to 1 week later (figure 1 ). The Malawi tuberculosis diagnostic algorithm recommends use of broad-spectrum antibiotics as trial-of-antibiotics after negative sputum tests are provided to patients who remain symptomatic. Therefore, the ideal population for randomisation for this study are patients on who already have negative results for smear microscopy or Xpert. However, that may have ethical challenges considering the implications of withholding treatment (if randomised to reference arm) from a symptomatic patient who, according to guidelines, should be given antibiotics. The first visit therefore was the most ideal time for randomisation and is in line with recommendations for test interval in investigations evaluating diagnostic tests with respect to the time interval between the index test (trial-of-antibiotics) and the reference test (mycobacteriology sputum sample collection). The timing also conforms to common clinical practice of prescribing trial-of-antibiotics at the same time as sputum collection to reduce diagnostic delay. The design was discussed with the District Health Office and the National Tuberculosis Programme ahead of ethics submission.

\section{Known drug reactions}

Azithromycin and amoxicillin have a long registration history, have been widely used globally and are well tolerated. Rare side effects for azithromycin include nervousness, dermatological reactions including Stevens-Johnson syndrome, anaphylaxis and prolonged QT interval. Rare side effects for amoxicillin are mental state changes, lightheadedness, photosensitivity and severe allergic reactions.

\section{Concomitant medication and interaction with other therapies}

We do not have any restrictions with respect to concomitant medications apart from those listed in the exclusion criteria. We expect some participants to be on HIV antiretroviral drugs and some to subsequently start tuberculosis therapy. Important interactions therefore would be those between the product and HIV antiretroviral drugs. There is no moderate or major interaction between either azithromycin or amoxicillin with the classes of HIV antiretroviral drugs currently used in Malawi. 


\section{Trial restrictions}

We do not require participants to have any dietary restrictions. We will also accept co-administration with contraception. Azithromycin and amoxicillin are both considered safe in pregnancy, so we will include pregnant women should they be eligible.

\section{Assessment of compliance}

On Day 8, we will document self-reported compliance adherence of study products.

\section{Withdraw of interventions}

The investigator may also terminate a participant from study product if indicated by an adverse reaction. If a participant stops taking study product either voluntarily or by investigator decision, they will be encouraged to remain in follow-up and their data will form part of intention-to-treat analyses.

\section{Study outcomes}

The clinical trial has two separately powered, and distinctly assessed primary outcomes, one for diagnostic evaluation (Primary outcome 1: Day 8) and the other for clinical impact (Primary outcome 2: Day 29) of the intervention. The following are descriptions of all study outcomes:

\section{Primary outcome 1: specificity of day 8 symptom change versus mycobacteriology}

The first primary outcome is the proportion of patients without tuberculosis (by sputum tests) who report improvement of their baseline illness when asked 7 days after randomisation (Day 8 study visit). This outcome can be thought of as diagnostic specificity if you take sputum test results as a reference standard and change in symptoms at Day 8 as the investigational test (figure 3). In this case the possible results of the investigational test are improvement and no improvemet (no change or worsened) in response to the question: on Day 1, you reported that you were unwell; compared with that day, has your illness worsened, remained the same or improved?

As with all self-rated outcomes, social desirability bias (tendency of participants to answer questions in

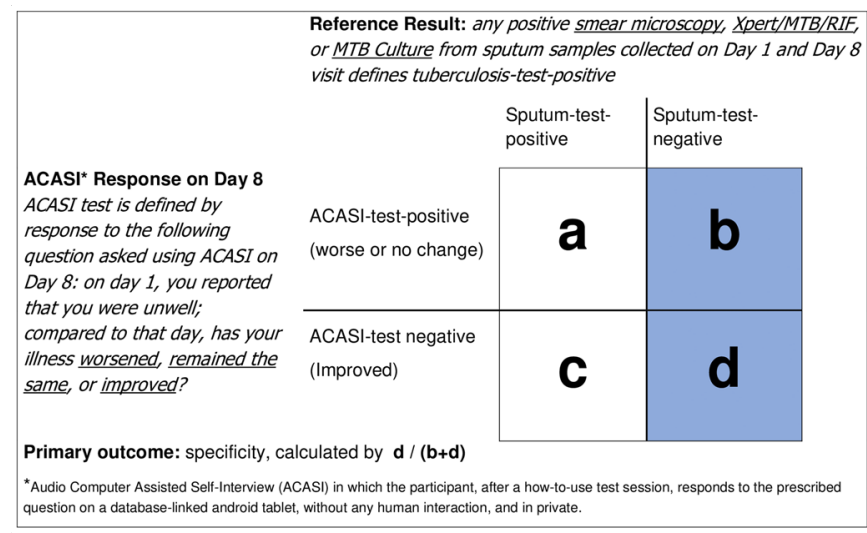

Figure 3 Assessing the diagnostic value of a change in symptoms from baseline to day 8. MTB,Mycobacterium tuberculosis; RIF, rifampicin. a manner that will be viewed favourably by healthcare worker), and interviewer bias (interviewers' subconscious or conscious influencing subject response) may affect the outcome. To minimise these biasses in evaluation of improvement of baseline symptoms the interview will be conducted using audio computer-assisted self-interview (ACASI), a platform that allows patients to report their health state in private and directly into a database via an audio questionnaire administered by a tablet. The lack of human-to-human interaction will minimise interviewer, ascertainment and social desirability biasses. Another concern with open-label design is placebo-effect favouring those randomised to antibiotics over the standard of care arm that is however not addressed in our design.

We developed, piloted and optimised the ACASI questionnaire in the study target population and arrived at the question: on Day 1, you reported that you were unwell; compared with that day, has your illness worsened, remained the same or improved? Before proceeding to the self-interview, participants will be oriented using test questions until study staff are sure that they will be able to go through the interview on their own. We will term ACASI interview outcome as ACASI-test-negative if the participant reports improvement or ACASI-test-positive if the participant reports no change or worsening (figure 3 ).

The mycobacteriology reference standard will be defined in participants with at least one valid sputum test result on Days 1 and 8 as sputum-test-positive if there is at least one positive of smear microscopy, Xpert MTB/ RIF or MTB culture; and as sputum-test-negative if none of the tests is positive. To minimise bias, the sputum tests will be performed by a high-quality research laboratory in the University of Malawi College of Medicine by staff with no access to participant treatment allocation information or symptom results.

The specificity of Day 8 symptom change (the index test measured using ACASI) against mycobacteriology tests (reference test) is defined as: proportion of sputumtest-negative who are ACASI-test-negative.

\section{Primary outcome 2: clinical impact of trial-of-antibiotics}

We will investigate the overall clinical impact of trial-ofantibiotics by comparing the Day 29 risk of any of death, hospitalisation and 'missed tuberculosis' (untreated mycobacteriological or radiological tuberculosis). All these events can lead to mortality and are potential consequences of trial-of-antibiotics; therefore, grouping them as a composite endpoint appropriately represents the effect of the intervention because: (1) there are similarities in the importance of each of the components, (2) the components occur with similar frequencies in the patient population and (3) the direction of effect is anticipated to be the same for all. ${ }^{22}$

The connection between trial-of-antibiotics and risk of hospitalisation and death assumes a protective effect of antibiotics. In patients presenting with chronic cough at primary care in high HIV prevalence settings, frequencies 
of mortality and hospitalisation over a 2 months period are similar, ranging from $2 \%$ to $6 \%{ }^{23}$

We have included missed tuberculosis diagnosis in our composite clinical outcome because this too can lead to death. We are defining 'missed tuberculosis' as participants who meet standard mycobacteriological and radiological tuberculosis definitions but are incorrectly classified as tuberculosis-negative and not yet on tuberculosis treatment by Day 29. Clinical, radiological and microbiological evaluation for tuberculosis will be done at Day 8, Day 29, as well as day between these two for patients who report worsening symptoms.

\section{Secondary outcome 1: impact of trial-of-antibiotics on antimicrobial resistance}

We will use Streptococcus pneumoniae isolated from swabs of the nasopharynx as the indicator pathogen for AMR evaluation. An ecological niche for many bacterial species, the upper respiratory tract also presents a convenient window for investigating antimicrobial resistance. S. pneumoniae is the organism of choice not only for being an important cause of respiratory tract infections but also because it often colonises the upper respiratory tract, acquires resistance readily and has well documented laboratory investigation procedures in place. ${ }^{24}$

We will define AMR positive as having nasopharyngeal isolates of $S$. pneumoniae that are resistant to any of the following commonly used antibiotics: ceftriaxone, amoxycillin, cefoxitin, azithromycin and erythromycin as determined using disc diffusion technique; and AMR negative as either (1) not isolating any $S$. pneumoniae or (2) isolating any $S$. pneumoniae that is not resistant to any of the assessed antibiotics. For each arm, and at both baseline and Day 29, we will report proportion of AMR positive participants. The study outcome will be the proportion of AMR positive participants at Day 29.

Secondary outcome 2: diagnostic value of trial-of-antibiotics in all patients including those without a valid sputum result

In this analysis, all will remain as described for primary outcome 1 except for the denominator, which will now include those without a valid sputum test result. The mycobacteriology reference standard for secondary outcome 2 will be defined as sputum test positive if at least one positive of smear microscopy, Xpert MTB/ RIF or MTB culture from samples collected on Days 1 and 8 . The reference test will be sputum-test-negative if none of the tests is positive and where there is no valid sputum test result available. The most likely reason for not having a valid sputum result will be inability to produce sputum, but other explanations will be: lost sample before laboratory analysis, an invalid laboratory reading or contamination. We have opted to analyse this population because in symptomatic adults of the study setting, failure to produce sputum can be as high as $13 \% .^{23}$
Secondary outcome 3: economic evaluation

The objective of the economic evaluation is to undertake a cost-utility analysis to estimate the incremental costeffectiveness of trial-of-antibiotics using azithromycin and trial-of-antibiotics using amoxicillin in comparison to standard of care, and to each other. We will systematically compare costs and consequences associated with the interventions. We will perform a within trial comparison of the three treatment arms to estimate the incremental cost per quality-adjusted life year (QALY) gained for the azithromycin or amoxicillin arm in comparison to standard of care. Costs will be estimated from the Malawian Ministry of Health perspective. Health outcomes will be quantified in QALYs, estimated from participants' responses to the Chichewa version of the EQ-5D-3L, a health-related quality of life measure. ${ }^{25}{ }^{26}$ We will adopt a time horizon matching the length of participant follow-up to achieve the within trial evaluation.

\section{Exploratory outcomes}

Our exploratory analyses will be comparisons between the azithromycin and amoxicillin arms for all our primary and secondary outcomes.

\section{Planned subgroup analyses}

We will perform analysis of primary outcomes stratified by HIV status and by antiretroviral therapy (ART) status as documented on enrolment day. This is important because the study site has high prevalence of HIV and associated bacterial infections which may be amenable to antibiotics used for trial-of-antibiotics.

\section{Study procedures}

Figure 2 and table 1 presents the study time schedule including a summary of patient identification, baseline procedures and outcome ascertainment at Day 8 and Day 29 follow-up visits.

\section{Screening}

Study staff will approach patients with symptoms of pulmonary tuberculosis (including cough of any duration, fever, weight loss and night sweats) with information about the study and seek written informed consent (online supplementary appendix 4) from all patients who meet eligibility criteria. After consenting, a participant will be given a unique study identification number confirming enrolment.

\section{Randomisation}

Randomisation will be in the ratio $1: 1: 1$ to the three arms of the trial, using block-randomisation with variable block sizes, and stratified by study site. An independent statistician will prepare the randomisation list using ralloc command in Stata software, then print each allocation alongside a randomisation number and seal in opaque envelopes. On confirming eligibility and consenting status a designated site staff will open the next available sequentially numbered randomisation envelopes and administer the allocated study arm. 
Table 1 Key study procedures over the study period

\begin{tabular}{|c|c|c|c|}
\hline \multirow[b]{3}{*}{ Time point } & \multicolumn{3}{|c|}{ Study period } \\
\hline & \multirow{2}{*}{$\begin{array}{l}\text { Enrolment } \\
\text { Day } 1\end{array}$} & \multicolumn{2}{|c|}{ Follow-up } \\
\hline & & Day 8 & Day 29 \\
\hline \multicolumn{4}{|l|}{ Enrolment } \\
\hline Eligibility screen & $x$ & & \\
\hline Informed consent & $x$ & & \\
\hline Allocation & $x$ & & \\
\hline \multicolumn{4}{|l|}{ Interventions } \\
\hline Azithromycin & $x$ & & \\
\hline Amoxicillin & $\mathrm{x}$ & & \\
\hline Standard of care & $x$ & & \\
\hline \multicolumn{4}{|l|}{ Assessments } \\
\hline Demographics & $x$ & & \\
\hline History of antibiotic use & $x$ & $x$ & $x$ \\
\hline History and examination* & $x$ & $x$ & $x$ \\
\hline Sputum collection $†$ & $x$ & $x$ & \\
\hline Urine for TB LAM test $\neq$ & $x$ & $x$ & \\
\hline $\begin{array}{l}\text { Nasopharyngeal swab for } \\
\text { AMR§ }\end{array}$ & $x$ & & $x$ \\
\hline HIV test & $x$ & & \\
\hline Linking to routine care & $x$ & $x$ & $x$ \\
\hline ACASIף & & $x$ & \\
\hline Clinical events ${ }^{\star *}$ & & & $x$ \\
\hline Update contact \& address & & $x$ & $x$ \\
\hline
\end{tabular}

${ }^{*}$ For symptomatic participants, Day 8 sputum mycobacteriology should be fast-tracked to inform care before they leave the clinic. †Give sputum bottles at the end of Day 1 visit for submission on Day 8. Also collect sputum and perform mycobacteriology at any time of the study when clinically indicated.

łUrine lipoarabinomannan for tuberculosis diagnosis (TB LAM). §Nasopharyngeal swab for Streptococcus pneumoniae culture and sensitivity as a way of determining risk of antimicrobial resistance (AMR).

ๆAudio computer-assisted self-interview (ACASI) for documenting change of symptoms on Day 8 versus Day 1.

**|llnesses, clinic visits, radiological outcomes, new HIV diagnosis, new tuberculosis diagnosis, death, hospitalisation, missed tuberculosis diagnosis, HIV care loss to follow-up and tuberculosis care loss to follow-up.

\section{Blinding}

The study is not placebo controlled because of funding limitations, and so will not use blinding due to the nature of the study design. However, study team masking will be maintained with all study outcome assessment occurring without reference to randomisation arm.

\section{Baseline procedures}

At baseline, we will collect demographic data, clinical history, record vital signs, height and weight. Participants will be requested to provide two sputum samples for Xpert $\mathrm{MTB} / \mathrm{RIF}$ and two more sputum samples the following morning for smear microscopy and MTB culture. We will also collect a urine sample for lipoarabamannan antigen detection (TB LAM); and a nasopharyngeal swab for pneumococcal culture and sensitivity testing. We will offer and perform HIV testing according to the national algorithm, and link all who test positive to care. To minimise loss to follow-up, we will collect contact phone numbers, a physical address and geolocation information.

\section{Participant follow-up}

On Day 8 , the first activity (ahead of any other interaction with study staff) will be the ACASI. Other activities include providing results for Day 1 tuberculosis tests and linking those who test positive to care, collection of another sputum sample for smear microscopy and $\mathrm{Myco-}$ bacterium tuberculosis (MTB) culture and management of ongoing symptoms and other illnesses. On visit Day 29, the final study visit, we will document participant vital status, hospitalisations and establish adherence to HIV and tuberculosis treatment. We will also collect nasopharyngeal swab samples from all participants, and sputum from those with tuberculosis symptoms.

\section{Participant retention}

To minimise loss to follow-up, we will record geolocation information of participants' place of residence using ePAL android application, a high-resolution mapping system validated in Blantyre. We will also record up to three contact phone numbers of the participant and their nominated friends and relatives. We will not replace participants who discontinue study participation or study treatment regardless of reason for withdrawal or discontinuation or the time either of these occurs.

\section{Data management}

We will collect data using TeleForm (paper based system that uses optical character recognition) and Open Data Kit systems (ODK, an electronic data capture system installed on android devices). Data will be committed to a secure database located at Malawi-Liverpool Wellcome Trust within 2 days for TeleForm, and 7 days for ODK.

\section{Statistical approach}

We will summarise the processes of recruitment including non-eligibility and reasons of exclusion in a CONSORT (Consolidated Standards of Reporting Trials) flow chart. We will describe the study participants by their baseline characteristics, by arm. We will perform analyses of all our outcomes based on an intention-to-treat analysis (using the arm patient was randomised to). Analysis for primary outcome 1 will be restricted to participants with a valid sputum test result. We will report measures of effect from the following comparisons: (i) azithromycin or amoxicillin (combined) versus standard of care, (ii) azithromycin versus standard of care and (iii) amoxicillin versus standard of care.

We will use a generalised linear model (GLM) with identity link to estimate risks differences and the GLM with $\log$ link to estimate risk ratios for the three comparisons, adjusting for study site. For each comparison, we will 
report $95 \%$ CIs and $\mathrm{p}$ values from the likelihood test. If outcomes are rare, or the GLM model does not converge, we will use logistic regression to estimate the treatment effect using an OR. We will not perform adjustments for multiple comparisons but will report all effect sizes with their $95 \%$ CIs and $p$ values to facilitate appropriate interpretation of our results.

We will perform data cleaning and analysis using Stata release 15 (StataCorp, College Station, Texas, USA). The statistical approach will be expanded in a detailed statistical analysis plan, which will be finalised before unblinding the study data.

\section{Sample size and power}

We performed power and sample size estimations for the diagnostic impact, clinical impact and AMR impact outcomes as described below. Our sample size estimations are based on planned analysis that will use $\chi^{2}$ test for comparing two independent proportions.

\section{Diagnostic impact outcome}

We assume that at Day 8, change in well-being from baseline state in trial-of-antibiotics (azithromycin or amoxicillin) arms will correctly classify $60 \%$ of all mycobacteriology-negative participants (ie, $60 \%$ specificity of Day 8 symptom change in trial-of-antibiotics arms). ${ }^{12}$ We wanted to estimate a sample size that would provide a discriminatory power of $80 \%$ at a two-sided significance level of $5 \%$, to detect at least $10 \%$ difference in specificity (ie, $\leq 50 \%$ specificity of Day 8 symptom change in standard of care arm).

\section{Sample size for a combination of two antibiotic arms against standard of care arm}

The sample size estimates along with assumptions for this comparison are shown in the table 2A. To achieve the desired $80 \%$ discriminatory power, we will need to recruit at least 290 sputum-test-negative participants per arm. Accounting for TB prevalence, ability to produce and submit sputum, and loss to follow-up increases the sample to 453 per arm or 1359 for the whole study.

\section{Sample size for one antibiotic arm against standard of care arm}

The sample size estimates along with assumptions for this comparison are shown in the table 2B. To achieve the desired $80 \%$ discriminatory power, we will need to recruit at least 388 sputum-test-negative participants per arm. Accounting for TB prevalence, ability to produce and submit sputum, and loss to follow-up increases the sample to 606 per arm or 1819 for the whole study (The ethics approved protocol uses an older calculation that yields 625 per arm and 1875 for whole study).

\section{Power for clinical impact outcome}

For the clinical impact of trial-of-antibiotics outcome, we assume a $4 \%$ baseline risk of composite outcome, and a loss to follow-up of $10 \%$ by Day 29 . Using the sample size of 625 participants per arm (obtained in table 2B), and a
Table 2A Sample size estimation for the diagnostic impact outcome comparing a combination of two antibiotic arms to standard of care arm (2:1 comparison)

\begin{tabular}{|c|c|c|}
\hline $\begin{array}{l}\text { Power (X2 } \\
\text { difference between } \\
\text { independent } \\
\text { proportions) }\end{array}$ & $\begin{array}{l}\text { Effect size ( } 50 \% \text { SoC } \\
\text { vs } 60 \% \text { amoxycillin } \\
\text { or azithromycin) }\end{array}$ & $\begin{array}{l}\text { Effective } \\
\text { sample per } \\
\text { arm (sputum } \\
\text { negative } \\
\text { participants } \\
\text { needed) }\end{array}$ \\
\hline 0.80 & 0.10 & 290 \\
\hline 0.85 & 0.10 & 332 \\
\hline 0.90 & 0.10 & 388 \\
\hline
\end{tabular}

Highlighted entries indicates target power and respective sample size estimates based on knowledge of TB risk, ability to produce and submit sputum and loss to follow-up.

Stata code: power two proportions 0.5 and 0.6 , test $\left(\chi^{2}\right)$, power (0.80), $\mathrm{n}$ ratio (2).

SoC, standard of care; TB, tuberculosis.

Table 2B Sample size estimation for the diagnostic impact outcome one antibiotic arm to standard of care arm (pairwise comparison)

\begin{tabular}{|c|c|c|}
\hline $\begin{array}{l}\text { Power (X2 } \\
\text { difference between } \\
\text { independent } \\
\text { proportions) }\end{array}$ & $\begin{array}{l}\text { Effect size ( } 50 \% \text { SoC } \\
\text { vs } 60 \% \text { amoxycillin } \\
\text { or azithromycin) }\end{array}$ & $\begin{array}{l}\text { Effective } \\
\text { sample per } \\
\text { arm (sputum } \\
\text { negative } \\
\text { participants } \\
\text { needed) }\end{array}$ \\
\hline 0.80 & 0.10 & 388 \\
\hline 0.85 & 0.10 & 443 \\
\hline 0.90 & 0.10 & 519 \\
\hline
\end{tabular}

Highlighted entries indicates target power and respective sample size estimates based on knowledge of TB risk, ability to produce and submit sputum, and loss to follow-up.

Stata code: power two proportions 0.5 and 0.6 , test $\left(\chi^{2}\right)$, power (0.80), $\mathrm{n}$ ratio (1).

SoC, standard of care; TB, tuberculosis.

type I alpha of 5\%, we will be able to detect the difference between arms with $80 \%$ power, if the risk in the intervention arm is twice that of the standard of care arm. This estimate is applicable to all comparisons shown in section 3.

\section{Power for AMR outcome}

Study arms will be compared based proportion of participants with resistant $S$. pneumoniae on Day 29. We assume that $45 \%$ of Day 29 nasopharyngeal swabs will successfully grow $S$. pneumoniae, and that $10 \%$ of the isolates will meet the definition of resistance (described earlier under outcomes), and that $10 \%$ will be lost to follow-up by Day 29. Therefore, on Day 29, the standard of care arm (of 625 participants) will have $253 \mathrm{~S}$. pneumoniae isolates, 25 of which would meet the definition of resistance. This translates into a $4 \%(25 / 625)$ risk of AMR positive cases in the standard of care arm. To detect a two-fold change in odds of Day 29 AMR risk with at least $80 \%$ power, using Pearson's $\chi^{2}$ test, at 0.05 alpha, we will need at least 431 
and 553 participants per arm for the 2:1 and pairwise comparisons, respectively.

\section{Monitoring and oversight}

The trial will be monitored by the Research Support Centre Clinical Trials Unit of the University of Malawi College of Medicine. An independent Data and Safety Monitoring Board (DSMB), and a Trial Steering Committee have been set up and meet bi-annually.

\section{Trial closure}

We will consider the trial closed after completing follow-up of the last enrolled participant, and on recording all mycobacteriology laboratory reports. Antimicrobial resistance laboratory work will continue beyond trial closure. The trial may be terminated early by the Trial Steering Committee on recommendation of the DSMB. The halting rule for a trial arm is an unacceptable high level of deaths assessed using an alpha determined at the first DSMB meeting.

\section{PATIENT AND PUBLIC INVOLVEMENT}

Patients were involved in the design of the study especially the ACASI used for collecting primary outcome data. Health workers were involved in the design of study visits and patient flow.

\section{DISCUSSION}

The ACT-TB study will investigate the benefits and consequences of 'trial-of-antibiotics,' a widely promoted approach to many patients with suspected tuberculosis in low- and middle-income countries without solid evidence base. To our knowledge, ACT-TB study is the first RCT of this kind. Results of our trial will add to the evidencebase regarding routine diagnosis of tuberculosis in lowand middle-income countries and strengthen our fight against AMR. Both tuberculosis and AMR are diseases of major importance globally, with tuberculosis causing an estimated 1.6 million deaths in 2017 and AMR projected to cause 10 million deaths per year by $2050 .^{227}$

\section{Choice of study interventions}

We have chosen amoxicillin because it is the first-line treatment for outpatient management of pneumonia in Malawi and is commonly used for trial-of-antibiotics. It also provides data of immediate programmatic relevance and a starting point to investigate exacerbation of pre-existing AMR pressure. However, amoxicillin may not demonstrate the full benefits for trial-of-antibiotics because of organisms with intrinsic ('atypicals') or acquired (common in gram-negative organisms, and Staphylococcus aureus) penicillin resistance. ${ }^{28}$ Oral antibiotics that may provide the better diagnostic discrimination for bacterial versus mycobacterial causes of cough are macrolides, such as azithromycin, because of better intrinsic coverage of 'atypical' intracellular organisms such as mycoplasma species that cause community acquired pneumonia, ${ }^{29-31}$ and low levels of acquired macrolide-resistance in bacterial isolates in Malawi. ${ }^{28}$

\section{ACASI for post-treatment improvement assessment}

Our systematic review ${ }^{18}$ did not identify a consistent definition of tuberculosis or no tuberculosis based on trialof-antibiotics. A definition of clinical change following antibiotic treatment is necessary for the trial-of-antibiotics as this determines who get categorised as well or tuberculosis-positive. Approaches that ranged from selfreported improvement to a combination of clinical and radiological assessments are likely to be highly subjective and prone to bias, as well as being a potentially avoidable source of heterogeneity between studies. In this study, we hope to address these biasses (particularly, inter-observer variability, and patient/interviewer reporting or ascertainment biasses) by using self-rated change of illness (on Day 8) recorded using a self-completed questionnaire, the ACASI (described under outcomes). The ACASI questionnaire, the delivery platform and the resulting data management can all be replicated in future studies, creating potential for more standardisation in assessment of clinical response to treatment.

\section{Potential clinical impact of antibiotics}

In areas with high HIV prevalence, empirical antibiotics during tuberculosis investigations could be life-saving: mortality immediately before and after tuberculosis diagnosis is high, ${ }^{3} 32$ and is often secondary to severe bacterial infections. ${ }^{32-34}$ The leading aetiologies of infection and death on tuberculosis treatment as well as among outpatients with tuberculosis-like symptoms are $S$. pneumoniae and non-typhoidal salmonellae: both can present with cough (primary cause) or as comorbidities (superinfections) in patients presenting with active Mycobacterium tuberculosis disease ${ }^{32-34}$ If effective treatment of this type of life-threatening primary/super-infections reduces mortality during the diagnostic workup of suspected tuberculosis in people living with HIV, then empirical use of broad-spectrum antibiotics would be indicated for this purpose alone, irrespective of any diagnostic contribution to tuberculosis treatment decisions. In this context, azithromycin may be the most effective arm, as salmonella infections are highly sensitive to azithromycin, but not to amoxicillin. ${ }^{28}$

\section{AMR and trial-of-antibiotics}

Antimicrobial resistance relating to antibiotic use during evaluation for suspected tuberculosis has not been investigated before. Previous work has shown that empirical antibiotics can drive rapid emergence of antimicrobial resistance. ${ }^{35}{ }^{36}$ Co-trimoxazole prophylaxis for HIV-positive patients, introduced in 2005, was followed by near-universal resistance in bloodstream infections by $2010 .^{37}$ Mass drug administration of azithromycin for trachoma control initially reduces nasopharyngeal carriage of $S$. pneumoniae, but with increased macrolideresistance 6 months later. ${ }^{38} 39$ 
In this study we have the opportunity to assess the extent to which brief exposure drives antimicrobial resistance during diagnostic workup for tuberculosis. An ecological niche for many bacterial species, the upper respiratory tract also presents a convenient sampling opportunity for investigating antimicrobial resistance. ${ }^{40} S$. pneumoniae is the organism of choice not only for being an important cause of respiratory tract infections but also because it often colonises the upper respiratory tract, acquires resistance readily and has well documented laboratory investigation procedures in place. ${ }^{24}$ As exploratory analyses, we will also assess nasopharygeal colonisation and antimicrobial resistance in relation to tuberculosis treatment and HIV status.

\section{Important subgroups}

Clinical response to trial-of-antibiotics is possible and indeed well-described in patients with bacteriologically confirmed tuberculosis (ie, false-negatives/low sensitivity from the perspective of tuberculosis diagnosis) may relate to multiple super-infections. ${ }^{43}$ As such, this phenomenon may vary by HIV status, since multiple concurrent infections are a hallmark of advanced HIV immunosuppression, and are most commonly reported in patients with suspected tuberculosis in the pre-ART era. In 2015, in Malawi, $45 \%$ of adults who presented to primary care with prolonged cough ( $\geq 2$ weeks) were HIV-positive, of whom only 20\% started tuberculosis treatment on the basis of positive mycobacteriology. ${ }^{23}$ As such, the benefits and consequences of trial-of-antibiotics may vary by HIV status and ART coverage, and by subsequent tuberculosis treatment decisions. We will, therefore, include a prespecified subanalysis of trial outcomes stratified by HIV and ART status.

\section{Limitations}

The study has several limitations. First, we did not use a placebo-control arm. Second, the study is not adequately powered to evaluate safety of deferred antibiotic treatment or conduct subgroup analyses of outcomes by HIV status, both of which are important evidence gaps. Other limitations include the possibility that participants randomised to the standard-of-care arm may find alternative access to antibiotics therefore misclassifying exposure/intervention status. There is also a possibility of misclassifying active tuberculosis status because of the suboptimal nature of the available tests.

\section{ETHICS AND DISSEMINATION}

The study has been reviewed and approved by the University of Malawi College of Medicine Research and Ethics Committee (COMREC; registration number P.04/18/2381), the London School of Hygiene \& Tropical Medicine Research Ethics Committee (LSHTM EC; registration number 15232) and Regional Committee for Health and Research Ethics, NTNU-Midt, Norway (REK nord; registration number 208/1964). Regulatory approval has been granted by the Malawi Pharmacy, Medicines and Poisons Board (PMPB; registration number CTRC/III/14062018102). We will present any future protocol modifications to these bodies before implementing. We will submit results for publication in a peer-reviewed journal. We will submit abstracts to relevant national and international conferences. This work will also form part of a PhD thesis for THD, which he will submit to the LSHTM. This study will follow the standards set by CONSORT guidelines.

\section{Author affiliations}

${ }^{1}$ TB Centre, London School of Hygiene and Tropical Medicine, London, UK ${ }^{2}$ Helse Nord Tuberculosis Initiative, University of Malawi College of Medicine, Blantyre, Malawi

${ }^{3}$ Malawi-Liverpool Wellcome Trust Clinical Research Programme, University of Malawi College of Medicine, Blantyre, Malawi

${ }^{4}$ School of Public Health, University of the Witwatersrand, JohannesburgBraamfontein, Gauteng, South Africa

${ }^{5}$ School of Medicine, University of Saint Andrews, Saint Andrews, UK

${ }^{6}$ Institute of Infection and Global Health, University of Liverpool Faculty of Health and Life Sciences, Liverpool, UK

${ }^{7}$ Clinical Sciences, Liverpool School of Tropical Medicine, Liverpool, UK

\section{Twitter Titus Henry Divala @TDivala}

Contributors THD, KF and ELC are the main contributors to the conception, and design of the study. DS, MN and PM contributed to the general study planning and clinical design. NF contributed to the general study planning and antimicrobial resistance design. CK, LC, SC, and MJN contributed to the design, piloting and refining of study and clinical procedures. THD developed the first draft of the manuscript. All authors carefully reviewed and substantially contributed to the development of the trial protocol and this manuscript. All authors read and approved the final manuscript. THD is the guarantor for this work.

Funding The clinical trial is funded by the Commonwealth Scholarship Commission and the Helse Nord RHF grant awarded to THD. This work is part of THD's PhD work at London School of Hygiene \& Tropical Medicine (LSHTM). LSHTM is the sponsor of this clinical trial (sponsor address: Keppel Street, Bloomsbury, London WC1E 7HT). ELC is funded by a Wellcome Trust Senior Research Fellowship in Clinical Science: WT200901.

Competing interests None declared.

Patient and public involvement Patients and/or the public were involved in the design, or conduct, or reporting or dissemination plans of this research. Refer to the Methods section for further details.

Patient consent for publication Not required.

Provenance and peer review Not commissioned; externally peer reviewed.

Open access This is an open access article distributed in accordance with the Creative Commons Attribution Non Commercial (CC BY-NC 4.0) license, which permits others to distribute, remix, adapt, build upon this work non-commercially, and license their derivative works on different terms, provided the original work is properly cited, appropriate credit is given, any changes made indicated, and the use is non-commercial. See: http://creativecommons.org/licenses/by-nc/4.0/.

ORCID iD

Titus Henry Divala http://orcid.org/0000-0003-3029-9579

\section{REFERENCES}

1 Raviglione M, Sulis G. Tuberculosis 2015: burden, challenges and strategy for control and elimination. Infect Dis Rep 2016;8:6570.

2 World Health Organization. Global tuberculosis report 2018. World Health Organization, 2018.

3 Nliwasa M, MacPherson P, Mukaka M, et al. High mortality and prevalence of HIV and tuberculosis in adults with chronic cough in Malawi: a cohort study. Int J Tuberc Lung Dis 2016;20:202-10.

4 Getahun H, Harrington M, O'Brien R, et al. Diagnosis of smearnegative pulmonary tuberculosis in people with HIV infection or AIDS 
in resource-constrained settings: informing urgent policy changes. The Lancet 2007;369:2042-9.

5 Corbett EL, MacPherson P. Tuberculosis screening in high human immunodeficiency virus prevalence settings: turning promise into reality. Int J Tuberc Lung Dis 2013;17:1125-38.

6 Siddiqi K, Lambert M-L, Walley J. Clinical diagnosis of smearnegative pulmonary tuberculosis in low-income countries: the current evidence. Lancet Infect Dis 2003;3:288-96.

7 Ghana Health Service. Guidelines for the clinical management of TB and HIV co-infection in Ghana. Accra: Department DCaP, 2007.

8 Ministry of Health. Malawi national TB programme manual. 8 edn. Lilongwe: Programme NT, 2016.

9 National Department of Health. South Africa national tuberculosis management guidelines 2014. Pretoria: Coordination TDS, 2014.

10 World Health Organization. Global tuberculosis report 2016, 2016.

11 McDowell A, Pai M. Treatment as diagnosis and diagnosis as treatment: empirical management of presumptive tuberculosis in India. Int J Tuberc Lung Dis 2016;20:536-43.

12 Wilkinson D, Newman W, Reid A, et al. Trial-of-antibiotic algorithm for the diagnosis of tuberculosis in a district hospital in a developing country with high HIV prevalence. Int J Tuberc Lung Dis 2000;4:513-8.

13 Holmes AH, Moore LSP, Sundsfjord A, et al. Understanding the mechanisms and drivers of antimicrobial resistance. Lancet 2016;387:176-87.

14 World Health Organization. The evolving threat of antimicrobial resistance: options for action. Geneva: World Health Organization, 2012.

15 World Health Organization. Antimicrobial resistance: global report on surveillance. World Health Organization, 2014.

16 Laxminarayan R, Matsoso P, Pant S, et al. Access to effective antimicrobials: a worldwide challenge. Lancet 2016;387:168-75.

17 Divala TH, Fielding KL, Nliwasa M, et al. Sensitivity and specificity of using trial-of-antibiotics versus sputum mycobacteriology for diagnosis of tuberculosis: protocol for a systematic literature review. Syst Rev 2018;7:141.

18 Divala TH, Nliwasa M, Gupta-Wrigh A, et al. Is the current practice of using response to empirical broad-spectrum antibiotic treatment as an exclusion diagnostic for tuberculosis supported by evidence? systematic review and meta-analysis. 49th world conference on lung health of the International Union against tuberculosis and lung disease (the Union). Int J Tuberc Lung Dis 2018.

19 World Health Organization. High priority target product profiles for new tuberculosis diagnostics: report of a consensus meeting. Geneva, Switzerland: World Health Organization, 2014: 28-9.

20 National Tuberculosis Control programme. Malawi nationwide tuberculosis prevalence survey. 45th Union World Conference on Lung Health, Barcelona, Spain, 2014.

21 Joint Formulary Committee. British National formulary (BNF) 78. Pharmaceutical Press, 2019.

22 Montori VM, Permanyer-Miralda G, Ferreira-González I, et al. Validity of composite end points in clinical trials. BMJ 2005;330:594-6.

23 Nliwasa M, MacPherson P, Chisala P, et al. The sensitivity and specificity of loop-mediated isothermal amplification (lamp) assay for tuberculosis diagnosis in adults with chronic cough in Malawi. PLOS One 2016:11:e0155101.

24 Satzke C, Turner P, Virolainen-Julkunen A, et al. Standard method for detecting upper respiratory carriage of Streptococcus pneumoniae: updated recommendations from the world Health organization pneumococcal carriage Working group. Vaccine 2013;32:165-79.

25 EuroQol Group. EuroQol--a new facility for the measurement of health-related quality of life. Health Policy 1990;16:199-208.

26 Maheswaran H, Petrou S, MacPherson P, et al. Cost and quality of life analysis of HIV self-testing and facility-based HIV testing and counselling in Blantyre, Malawi. BMC Med 2016;14:34.

27 O'Neill J. Tackling drug-resistant infections globally: final report and recommendations. UK: HM Government and Welcome Trust, 2016.

28 Musicha P, Cornick JE, Bar-Zeev N, et al. Trends in antimicrobial resistance in bloodstream infection isolates at a large urban hospital in Malawi (1998-2016): a surveillance study. Lancet Infect Dis 2017;17:1042-52.

29 Lubell Y, Turner P, Ashley EA, et al. Susceptibility of bacterial isolates from community-acquired infections in sub-Saharan Africa and Asia to macrolide antibiotics. Trop Med Int Health 2011;16:1192-205.

30 Phua J, Dean NC, Guo Q, et al. Severe community-acquired pneumonia: timely management measures in the first 24 hours. Crit Care 2016;20:237.

31 Lim WS, Smith DL, Wise MP, et al. British thoracic Society community acquired pneumonia guideline and the NICE pneumonia guideline: how they fit together. Thorax 2015;70:698-700.

32 Cain KP, Anekthananon T, Burapat C, et al. Causes of death in HIVinfected persons who have tuberculosis, Thailand. Emerg Infect Dis 2009;15:258-64.

33 Bedell RA, Anderson STB, van Lettow M, et al. High prevalence of tuberculosis and serious bloodstream infections in ambulatory individuals presenting for antiretroviral therapy in Malawi. PLoS One 2012; $7:$ e39347.

34 Schleicher GK, Feldman C. Dual infection with streptococcus pneumoniae and mycobacterium tuberculosis in HIV-seropositive patients with community acquired pneumonia. Int $J$ Tuberc Lung Dis 2003;7:1207-8

35 Levy SB, Marshall B. Antibacterial resistance worldwide: causes, challenges and responses. Nat Med 2004;10:S122-9.

36 Goossens H, Ferech M, Vander Stichele R, et al. Outpatient antibiotic use in Europe and association with resistance: a cross-national database study. Lancet 2005;365:579-87.

37 Everett DB, Mukaka M, Denis B, et al. Ten years of surveillance for invasive streptococcus pneumoniae during the era of antiretroviral scale-up and cotrimoxazole prophylaxis in Malawi. PLoS One 2011;6:e17765

38 Coles CL, Mabula K, Seidman JC, et al. Mass distribution of azithromycin for trachoma control is associated with increased risk of azithromycin-resistant streptococcus pneumoniae carriage in young children 6 months after treatment. Clin Infect Dis 2013;56:1519-26.

39 Mitjà $\mathrm{O}$, Houinei $\mathrm{W}$, Moses $\mathrm{P}$, et al. Mass treatment with single-dose azithromycin for yaws. N Engl J Med 2015;372:703-10.

40 Man WH, de Steenhuijsen Piters WAA, Bogaert D. The microbiota of the respiratory tract: gatekeeper to respiratory health. Nat Rev Microbiol 2017;15:259-70. 